\title{
Perda de massa de flores de capuchinha após armazenamento
}

\author{
Andréia Sangalli; Silvana de Paula Quintão Scalon²; José Carlos Lopes de Carvalho ${ }^{3}$ \\ 1UEMS, Depto. Ciências Biológicas, C. Postal 351, 79804-970 Dourados-MS; ${ }^{2}$ UFGD, FCA, C. Postal 533, Dourados-MS; ${ }^{3}$ UEMS, \\ Depto. Química; sscalon@ceud.ufms.br
}

\section{RESUMO}

Avaliou-se a perda de massa pós-colheita de flores de capuchinha, em experimento conduzido no Laboratório de Bioquímica da UFMS, em setembro de 2004. Flores das cores vermelha e amarela foram imersas durante trinta minutos em água, ácido ascórbico a 2\% e 4\%, cloreto de cálcio a $2 \%$ e $5 \%$ e sacarose a $5 \%$ e $10 \%$, e armazenadas com e sem o uso de filme de PVC em câmara fria a $5^{\circ} \mathrm{C} \pm 2^{\circ} \mathrm{C}$. Foi analisada a perda de massa fresca, utilizando-se o delineamento inteiramente casualizado em fatorial $2 \times 2 \times 7$, com três repetições (8 flores por bandeja) e as médias comparadas pelo teste de Tukey, a 5\% de significância. A longevidade média das flores de capuchinha foi de oito dias para as flores acondicionadas na embalagem de PVC e armazenadas em câmara fria, enquanto as flores não acondicionadas apresentaram-se murchas a partir do segundo dia. Os tratamentos químicos, nas concentrações estudadas, não beneficiaram a conservação pós-colheita das flores da capuchinha.

Palavras-chave: Tropaeolum majus L., ácido ascórbico, sacarose, cloreto de cálcio, embalagem de PVC, conservação pós-colheita.

\section{ABSTRACT \\ Loss of nasturtium flower mass after storage}

The post-harvest behavior of nasturtium flowers was evaluated using sucrose, ascorbic acid and calcium chloride which were associated or not with polyethylene pack and stored under refrigeration. This work was carried out in a biochemical laboratory, in Dourados, Mato Grosso do Sul State, Brazil. Red and yellow flowers were immersed during thirty minutes in water, ascorbic acid at $2 \%$ and $4 \%$, calcium chloride at $2 \%$ and $5 \%$ and sucrose at $5 \%$ and $10 \%$, and then stored with and without package in a refrigerated chamber at $5^{\circ} \mathrm{C} \pm 2^{\circ} \mathrm{C}$. The trial was carried out in a complete randomized experimental design in a $2 \times 2 \times 7$ factorial scheme with three replications (8 flowers/tray). Nasturtium flowers stored with package in refrigerated chamber last for eight days, while flowers kept at environmental conditions withered after the second day. Treatments with ascorbic acid, calcium chloride and sucrose did not benefit post-harvest conservation of nasturtium flowers.

Keywords: Tropaeolum majus L. ascorbic acid, sucrose, calcium chloride, polyethylene package.

(Recebido para publicação em 20 de abril de 2006; aceito em 2 de agosto de 2007)

Tropaeolum majus L. semi-perene que apresenta arquitetura herbácea, com flores em forma de campânula, axilares, zigomorfas, cíclicas e hermafroditas. Popularmente é conhecida por capuchinha, chaguinha, alcaparra-de-pobre, chagas, mastruçodo-peru, papagaios, flor-de-sangue, agrião-do-méxico e capuchinha grande (Corrêa, 1926; Font Quer, 1993; Dematti \& Coan, 1999). Quanto às indicações medicinais, é citada como antiespasmódica, antiescorbútica, antisséptica, estimulante do bulbo capilar, expectorante, desinfectante das vias urinárias, digestiva, purgativa, dermatológica e algumas espécies do gênero também são usadas como anticoncepcional e no alívio dos sintomas de resfriados (Dematti \& Coan, 1999; Font Quer, 1993; Johns et al., 1982; Corrêa, 1926). As folhas têm grande quantidade de vitamina $\mathrm{C}$ e sais minerais e por isso é considerada hortaliça nutritiva, sendo suas folhas e flores utilizadas em saladas e sanduíches (Zurlo \& Brandão, 1989; Castellani, 1997).
As flores em geral são classificadas como produtos altamente perecíveis, pela natureza efêmera dos diferentes tecidos que as formam, pela alta atividade respiratória e pelo reduzido conteúdo de carboidratos de reserva (Nowak \& Rudnicki, 1990). Portanto, a comercialização destas flores, como flor de corte para fins ornamentais ou para fins alimentares, é limitada por sua curta longevidade. A baixa temperatura no armazenamento é um fator importante para o retardamento da deterioração, uma vez que diminui os processos metabólicos (transpiração e respiração) e o crescimento de patógenos, mantendo a qualidade por mais tempo e prolongando a vida pós-colheita de plantas e flores durante o período de armazenamento (Corbineau, 1992).

Atualmente, pesquisas vêm sendo desenvolvidas com o objetivo de prolongar a vida útil de flores. Dentre os tratamentos estudados, o suprimento adequado de carboidratos em flores, principalmente na forma de sacarose, além de fornecer energia para a manutenção da respiração vital, atua como regulador osmótico dos tecidos e é eficaz no aumento da longevidade de diversas espécies (Carneiro et al., 2002). Em flores de Lisianthus (Eustoma grandiflorum), a imersão em sacarose por 24 horas foi benéfica, prolongando a durabilidade das hastes e da primeira flor em um dia, quando comparada às flores do controle (Fukasawa et al., 2004). Carneiro et al. (2002) constataram que o uso de sacarose não prolongou a longevidade média das flores de zínia (Zinnia elegans), mas a taxa de produção de etileno dessas flores foi inibida, favorecendo o atraso de sua senescência. Entretanto, em flores de esporinha (Consolida ajacis), o uso de sacarose por imersão não foi efetiva para prolongar a vida das flores, mas observou-se inibição na produção de etileno e aumento da produção de $\mathrm{CO}_{2}$, com diminuição da respiração. Portanto, a efetividade da sacarose para aumentar a longevidade das flores é altamente dependente da espécie, sendo esse tratamento mais efetivo no estímulo à abertura das flores e aumento da absorção de água pela flor (Finger et al., 2004). 
O cálcio é um mineral que se liga às substâncias pécticas, dando origem aos pectatos de cálcio, que mantêm a estrutura e as propriedades das membranas celulares e conferem estabilidade à parede celular. É possível que o cálcio previna ou retarde o aparecimento de algumas desordens fisiológicas pela manutenção do metabolismo normal (Vilas Boas, 2000). A aplicação de cálcio através de soluções aquosas de seus sais, como o cloreto de cálcio, tem sido utilizada para manter a textura e a firmeza dos tecidos vegetais e suprimir a taxa respiratória e a produção de etileno, retardando a senescência dos frutos, hortaliças e flores (Poovaiah, 1986; Chitarra, 2000).

Reações oxidativas que causam escurecimento, descoloração de pigmentos endógenos, perda ou mudança do sabor, mudança da textura e perda nutricional são fatores que diminuem o tempo de vida de prateleira de hortaliças e frutos. O ácido ascórbico é um dos principais antioxidantes utilizado na prevenção do escurecimento e do desencadeamento de outras reações oxidativas. Também atua como quelante de enzimas oxidativas (Chitarra, 1999). Além de ser seguro para o consumo humano, a aplicação de ácido ascórbico como conservante pode levar ao aumento do teor de vitamina C (Préstamo \& Manzano, 1993). Souza et al. (2004) verificaram que no processamento mínimo de manga, o ácido ascórbico exerce efeito benéfico, aumentando os teores de vitamina $\mathrm{C}$ e prolongando o tempo de armazenamento do produto quando associado a cloreto de cálcio. Resultados semelhantes também foram observados por Reis et al., 2004 quando trabalhou com a qualidade do minimilho minimamente processado.

Um aumento da vida útil do produto também pode ser obtido através do uso de atmosfera modificada. Nesse processo, a atmosfera é alterada pelo uso de filmes de polietileno, que se caracterizam por serem boa barreira ao vapor d'água e terem permeabilidade seletiva ao $\mathrm{O}_{2}$ e $\mathrm{CO}_{2}$. Esse tipo de filme permite que a concentração de $\mathrm{CO}_{2}$ proveniente da respiração aumente, e a concentração de $\mathrm{O}_{2}$ diminua, retardando a senescência e a diminuição da produ- ção de etileno e da taxa respiratória (Chitarra \& Prado, 2000).

A perda de massa é um dos fatores limitantes à vida útil de muitos produtos hortícolas e é resultado do tempo de armazenamento e da transpiração (Vila Boas, 2000). Essa perda está relacionada à perda de água, causa principal da deterioração, que além de resultar em perdas quantitativas, também prejudicam a aparência (murchamento e enrrugamento), a textura e a qualidade nutricional (Carvalho, 2000). A taxa de transpiração é influenciada por fatores internos tais como características morfológicas e anatômicas, relação superfície-volume e estádio de maturidade, além de fatores externos e ambientais, como temperatura, umidade relativa, movimento de ar e pressão atmosférica. Assim, as perdas por transpiração podem ser controladas pelo tratamento do produto com ceras, coberturas superficiais e embalagens em filmes poliméricos, assim como pela manipulação do ambiente, mantendo a umidade relativa e o controle da circulação de ar (Vila Boas, 2000).

O cálcio e a atmosfera modificada têm sido amplamente utilizados para prolongar a vida útil de frutos e hortaliças. O ácido ascórbico vem sendo utilizado na conservação de alimentos minimamente processados. Entretanto, não foram encontrados trabalhos de flores com a aplicação desses tratamentos na bibliografia consultada.

O presente trabalho teve como objetivo avaliar a perda de massa pós-colheita de flores de capuchinha tratadas com sacarose, ácido ascórbico e cloreto de cálcio e armazenadas com ou sem filme de PVC sob refrigeração.

\section{MATERIAL E MÉTODOS}

O trabalho foi conduzido em setembro de 2004. As flores de capuchinha cultivar Jewel, utilizadas no experimento, foram colhidas no Horto de Plantas Medicinais da UFMS, Dourados-MS, no período matutino.

Para a colheita foram selecionadas flores recém abertas das cores vermelha e amarela, sendo armazenadas em bandejas com água e imediatamente transportadas para o Laboratório de Bio- química da UFMS, para realização dos tratamentos.

As flores foram acondicionadas em recipientes contendo as soluções água, ácido ascórbico $2 \%$ e $4 \%$, cloreto de cálcio $2 \%$ e $5 \%$ e sacarose $5 \%$ e $10 \%$. Os pedicelos foram imersos nas soluções durante 30 minutos, a fim de ocorrer absorção pelas células e condução até as pétalas. As flores imersas em água foram utilizadas como testemunha. As flores tratadas foram dispostas em bandeja de isopor (total de 8 flores/bandeja) e mantidas com ou sem embalagem. A modificação da atmosfera foi conseguida embalando as bandejas com filme de PVC transparente, esticável, de $10 \mathrm{~mm}$ de espessura e marca comercial RoyalPak. Posteriormente, as bandejas foram pesadas e acondicionadas em câmara fria a $5^{\circ} \mathrm{C} \pm 2^{\circ} \mathrm{C}$, com UR de $90 \%$, onde permaneceram durante todo o experimento.

O delineamento estatístico utilizado foi inteiramente casualizado, em esquema fatorial 2 cores das flores (vermelha e amarela) x 2 condições de armazenamento (com ou sem filme de PVC) x 7 tratamentos (água, ácido ascórbico, cloreto de cálcio e sacarose), com três repetições, sendo cada parcela representada por uma bandeja. Os dados foram analisados pelo teste $\mathrm{F}$ e havendo significância, foram comparadas por Tukey ao nível de 5\%.

Analisou-se a perda de massa fresca, determinada pela diferença de massa das flores no momento de cada avaliação e da massa inicial, com resultados expressos em \%.

Para determinar a longevidade das flores considerou-se o número de dias decorridos da colheita até o murchamento das flores.

\section{RESULTADOS E DISCUSSÃO}

As flores da testemunha apresentaram menores perdas de massa quando comparadas com as tratadas (Tabela 1). As flores acondicionadas em embalagem apresentaram perda de massa duas vezes menor em relação às flores sem embalagem. Comparando-se as cores, observou-se que as flores vermelhas apresentaram menor perda. 
A longevidade média das flores de capuchinha acondicionadas na embalagem de PVC e mantidas em câmara fria a $5^{\circ} \mathrm{C} \pm 2^{\circ} \mathrm{C}$ foi de oito dias após a colheita, enquanto as não acondicionadas apresentaram-se murchas e sem condições de comercialização a partir do segundo dia (Figura 1).

Pré-teste realizado pelos autores, utilizando-se geladeira com temperatura média de $10^{\circ} \mathrm{C}$, possibilitou constatar que a capuchinha é extremamente sensível à temperatura e a umidade relativa, pois mesmo as flores embaladas com filme de PVC apresentaram-se inviáveis à comercialização a partir do segundo dia após a colheita. Além da perda de massa, as pétalas apresentaram mudança de coloração, tornando-se mais opacas e com manchas necróticas.

Não foi observada interação significativa entre os tratamentos químicos, as cores e o uso ou não de embalagem. Entretanto, observou-se interação significativa entre cores de flor e embalagem. A perda de massa pelas flores de capuchinha durante os oito dias de armazenamento apresentou crescimento linear, sendo observada perda de massa pelas flores não embaladas de $34,2 \%$ e 38,8\% para flores vermelhas e amarelas, respectivamente. A perda de massa após oito dias, para as flores embaladas, foi de 20,6\% para as vermelhas e 21,0\% para as amarelas. As flores vermelhas mostraram-se mais resistentes à perda de massa quando armazenadas sem a embalagem de PVC.

Segundo Hardenburg et al. (1986) a excessiva perda de peso pode limitar a longevidade da flor, uma vez que, flores que perdem $10 \%$ ou mais de seu peso são consideradas murchas e inaptas à comercialização. Porém, deve-se considerar que para as flores de capuchinha embaladas com o PVC, a perda de massa constatada não interferiu no aspecto visual, estando as flores túrgidas, com cores vivas e sem qualquer necrose.

Os tratamentos químicos não reduziram as perdas de massa das flores de capuchinha. Entretanto, o uso de sacarose na solução de condicionamento na concentração de 2 a $20 \%$ ou mais, é muito comum. O uso da sacarose prolongou a longevidade de flores de Gypsophila paniculata, ave do paraíso

Tabela 1. Média geral da perda de massa de flores de capuchinha (Tropaeolum majus L.), após 8 dias de armazenamento (average mass loss of nasturtium flowers after 8-day storing). Dourados, UFMS. 2004.

\begin{tabular}{lc}
\hline Tratamentos & Perda de massa (\%) \\
\hline Testemunha & $19,62 \mathrm{c}$ \\
Ácido ascórbico 2\% & $21,37 \mathrm{bc}$ \\
Ácido ascórbico 4\% & $21,55 \mathrm{bc}$ \\
CaCl2 2\% & $23,95 \mathrm{a}$ \\
CaCl2 5\% & $21,95 \mathrm{ab}$ \\
Sacarose 5\% & $20,94 \mathrm{bc}$ \\
Sacarose 10\% & $20,02 \mathrm{bc}$ \\
Com embalagem * & $13,60 \mathrm{~b}$ \\
Sem embalagem & $29,08 \mathrm{a}$ \\
Flor vermelha * & $20,31 \mathrm{~b}$ \\
Flor amarela & $22,38 \mathrm{a}$ \\
\hline CV = (\%) & $16,14 \%$ \\
\hline
\end{tabular}

Médias seguidas de pelo menos uma mesma letra minúscula na coluna não são estatisticamente diferentes entre si pelo teste $\mathrm{F} *$ e Tukey a 5\% de probabilidade (Means followed by the same small letter in the column did not differ from each other, Tukey, 5\%).
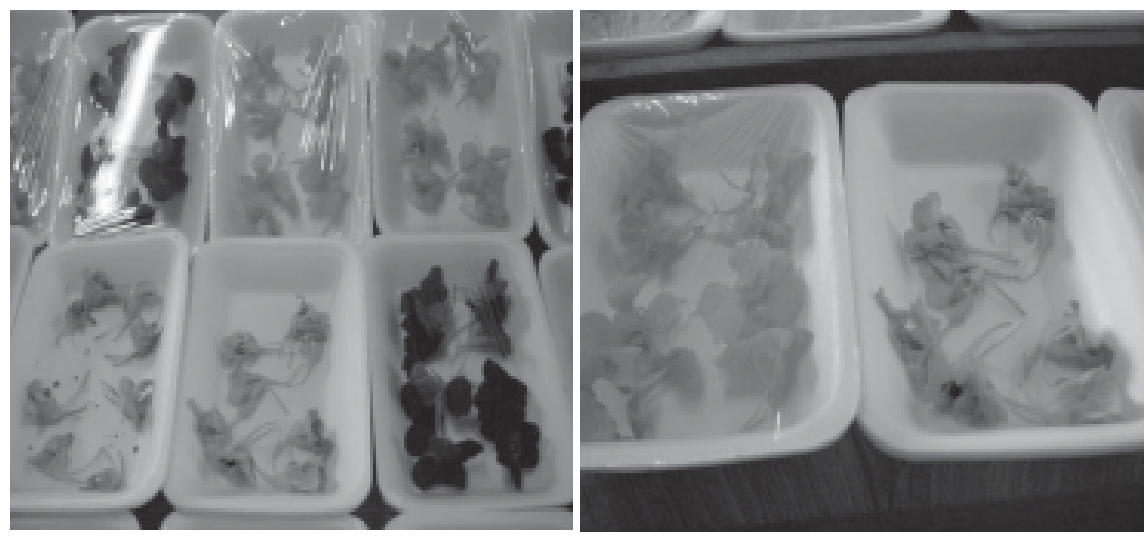

Figura 1. Aspecto das flores de capuchinha (Tropaeolum majus L.) após oito dias de armazenamento, com e sem o uso de embalagem (Appearance of nasturtium flowers after 8day storing). Dourados, UFMS. 2004.

(Strelitzia reginae) e zínia (Zinnia elegans) (Carneiro et al., 2002; Van Doorn \& Reid, 1992). Portanto, a efetividade da sacarose em elevar a longevidade das flores é altamente dependente da espécie, sendo este tratamento mais efetivo no estímulo à abertura das flores e aumento da absorção de água pela flor (Finger et al., 2004).

A embalagem provavelmente inibiu a produção de etileno das flores de capuchinha, retardando o processo respiratório e, conseqüentemente, a sua senescência. Além disso, diminuiu os processos metabólicos (transpiração e respiração) durante o período de armazenamento, proporcionando maior tempo de vida útil das flores de capuchinha.
O uso da embalagem de PVC, associada a baixas temperaturas foi fundamental para aumentar de dois para oito dias o tempo de vida útil das flores de capuchinha. A aplicação de cloreto de cálcio, ácido ascórbico ou sacarose nas concentrações estudadas não beneficiaram a conservação pós-colheita. A coloração da flor não interferiu na perda de massa, quando o armazenamento da mesma foi feito com a embalagem de PVC.

\section{REFERÊNCIAS}

CARNEIRO TF; FINGER FL; SANTOS VR; MELO NEVES LL; BARBOSA JG. 2002. Influência da sacarose e do corte da base da haste na longevidade de inflorescências de Zinnia elegans. Pesquisa Agropecuária Brasileira 37:. 
CARVALHO AV. 2000. Avaliação da qualidade de kiwis cv. "Haryward", minimamente processados. Dissertação - Universidade Federal de Lavras, Lavras, 2000.

CASTELLANI DC. 1997. Crescimento, anatomia e produção de ácido erúcico em Tropaeolum majus L. 1997. Dissertação - Universidade Federal de Viçosa, Viçosa.

CHITARRA AB. 1999. Armazenamento de frutos e hortaliças por refrigeração. Lavras: UFLA/FAEPE, 62p.

CHITARRA MIF. 2000. Processamento mínimo de frutos e hortaliças. Lavras: UFLA/FAEPE, 119p.

CHITARRAAB; PRADO MET. 2000. Utilização de atmosfera modificada e controlada em frutos e hortaliças. Lavras: UFLA/FAEPE, 66p.

CORBINEAU F. 1992. El enfriamiento de flores y plantas. Universidad de Pierre y Marie Curie, Paris y CNRS. Mendon, Francia, p.62-90.

CORRÊA MP. 1926. Dicionário das plantas úteis do Brasil e das exóticas cultivadas. Rio de Janeiro: Ministério da Agricultura e Instituto Brasileiro de Desenvolvimento Florestal. v. 1.

DEMATTI MESP; COAN RM. 2004. Jardins com plantas medicinais. Jaboticabal: FUNEP.

FINGER FL; CARNEIRO TF; BARBOSA JG 2004. Senescência pós-colheita de inflorescências de esporinha (Consolida ajacis). Pesquisa Agropecuária. Brasileira 39: 533-537.
FONT QUER P. 1993. Plantas medicinales: el dioscórides renovado. Barcelona: Editorial Labor S.A., Tomo II, p. 251-637.

FUKASAWA ST; CAMPOS DVA; WADA JF LASCHI D; KATZ I. 2004. Conservação e aumento da longevidade floral de Lisianthus. Horticultura Brasileira 22, Suplemento, CDROM. Trabalho apresentado no $44^{\circ}$ Congresso Brasileiro de Olericultura.

HALEVY AH; MAYAK S. 1979. Senescence and postharvest physiology of cut flowers: part 1. Horticultural Reviews 1: 79-80.

HARDENBURG RE, WATADAAE, WANG CY. 1986. The commercial storage of fruits, vegetables and florist and nursery stocks. Washington: U.S. Departament of Agriculture, 136 p. (Agriculture Handbook, $n^{\circ} 66$ ).

JOHNS T; KITTS WD; NEWSOME F; NEIL TOWERS GH. 1982. Anti-reproductive and other medicinal effects of Tropaeolum tuberosum L. Journal of Ethnopharmacology 5: 149-161.

NOWAK J; RUDNICKI RM. 1990. Postharvest handling and storage of cut flowers, florist greens, and potted plants. Portland: Timber, 210p.

POOVAIAH BW. 1986. Role of calcium in prolonging storage life of fruits and vegetables. Food Technology 40: 86-89.
PRÉSTAMO G; MANZANO P. 1993. Peroxidases of selected fruits and vegetables and the possible use of ascorbic acid as na antioxidant. HortScience 28: 48-50.

REIS KC; PEREIRA J; LIMA LCO. 2004. Efeito do lactato de cálcio e do ácido ascórbico sobre a qualidade do minimilho minimamente processado. In: ENCONTRO NACIONAL SOBRE PROCESSAMENTO MÍNIMO DE FRUTAS E HORTALIÇAS, 3., 2004, Viçosa. Palestras, resumos e oficinas...Viçosa: UFV, p.168.

SOUZA JF; SANTOS RM; YAGUIU P; CARVALHO MS; CARNELOSSI MAG; LIRA ML; VIÉGAS PRA. 2004. Utilização de ácido ascórbico e cloreto de cálcio na conservação de manga cv. Espada minimamente processada. In: ENCONTRO NACIONAL SOBRE PROCESSAMENTO MÍNIMO DE FRUTAS E HORTALIÇAS, 3., 2004, Viçosa. Palestras, resumos e oficinas...Viçosa: UFV, p.146.

VAN DOORN WG; REID MS. 1992. Role of ethylene in flower senescence of Gypsophila paniculata L. Postharvest Biology and Technology 1: 265-272.

VILAS BOAS EVB. 2000. Perdas pós-colheita. Lavras: UFLA/FAEPE, 64p.

ZURLO C; BRANDÃO M. 1989. As ervas comestíveis. Rio de Janeiro: Globo, 167p. 